\title{
La formación del docente para el uso de ambientes b-learning con el fin de preservar la dignidad humana y la ética mundial
}

\author{
José Eduardo Padilla Beltrán, Ph. D.* \\ Karolina González Guerreo, M. Sc.** \\ Diego Armando Rincón Caballero Lic. Tec.**
}

Resumen. El propósito de esta investigación es develar las funciones y roles del docente, quien se apoya del $b$-learning para orientar los aprendizajes de los estudiantes, con el fin de llevarlos a las Facultades de Educación, teniendo en cuenta la formación de los docentes con miras a preservar la dignidad humana y la ética mundial. El abordaje metodológico se realizó

* Licenciado en matemáticas, Magíster en Administración y Supervisión Educativa, Magíster en Educación con énfasis en Evaluación Educativa, Especialista en Orientación Educativa, Doctor en Educación. Docente de Planta de la Universidad Militar Nueva Granada, docente de la Facultad de Educación de la Universidad de San Buenaventura, sede Bogotá. Líder del Grupo de Investigación PYDES «Pedagogía y Didáctica de la Educación Superior».edopadilla@gmail.com

** Licenciada en Electrónica, Magíster en Educación. Docente de planta de la Universidad Militar Nueva Granada, directora del Centro de Investigaciones de la Facultad de Educación y Humanidades, editora de la revista Educación y Desarrollo Social. kgonzalezg@gmail.com

*** Licenciado en Diseño Tecnológico. Joven investigador de la Universidad Militar Nueva Granada. Diego.rincon@unimilitar.edu.co

ITINERARIO EDUCATIVO • AÑO XXV, N. ${ }^{\circ} 58$ • I65-I84 • JULIO-DICIEMBRE DE ZOII 
desde una perspectiva cualitativa, abierta y flexible apoyado del ATLAS.ti para la revisión de más de ciento veinte artículos. La investigación al momento permite determinar las funciones y roles de los maestros, quienes hacen uso de los ambientes virtuales para orientar sus saberes.

Palabras clave. Formación docente, b-learning, dignidad, pedagogía, humano, ética.

Abstract. The purpose of this research is to uncover the functions and roles of the teacher, who is supported on b-learning to guide student's learning, in order to bring it to the Education Faculties to be considered in the teachers training in order to preserve human dignity and global ethic. The methodological approach was made from a qualitative perspective, open and flexible and supported on ATLAS.ti to check out more than one hundred and twenty papers. The research at the time allows determining the functions and roles of teachers who are in training, those who use virtual environments to guide their knowledge.

Keywords. Teachers training, b-learning, dignity, pedagogy, human, ethics.

«Todo ser humano, sea hombre o mujer, blanco o negro, rico o pobre debe recibir un trato humano»

Hans Küng

\section{Introducción}

Con los avances en el desarrollo tecnológico de las plataformas (Moodle y Blackboard), aparecieron nuevas formas de mediación pedagógica y educativa como apoyo de la enseñanza en su 
modalidad presencial, a distancia o virtual. Debido a lo anterior, pronto en las instituciones educativas las clases presenciales, los módulos, los textos fueron siendo apoyadas o cambiados por aulas y bibliotecas virtuales, presentando un nuevo escenario laboral de los docentes, quienes ahora ameritan de habilidades y capacidades para desempeñarse en estos ámbitos.

Al comienzo, los docentes se apropiaron de algunos conocimientos sobre el manejo de las Mediaciones Tecnológicas de Información y Comunicación [MTIC] por iniciativa propia en forma autodidacta, pero como afirma Padilla J.E. «Sin embargo, queda claro que esta vía de autoaprendizaje deja sin descubrir un sinnúmero de procesos y procedimientos en el manejo acertado de las MTIC que no le permiten hacer uso eficiente de la herramienta» $(2011$, p.8). Y, en consecuencia, se hace necesario incluir en los currículos de los programas de formación de docentes estas nuevas áreas. Al mismo tiempo, cabe examinarse si con el uso de las MTIC, el docente preserva la dignidad humana de su aprendiz y la propende por la ética mundial.

$\mathrm{Al}$ revisar el concepto de dignidad humana se encuentra en notoria evolución. Para Pelé, A. (2005) se introduce más bien desde la indignidad en condiciones sociales, psicológicas donde se encuentran las personas y las comunidades; y por otro lado, en algunas ocasiones se relaciona con los procesos biotecnológicos desde la implicación de las nuevas tecnologías. Por ejemplo, en la época moderna, «la dignidad del hombre deriva de su naturaleza humana pero dicha naturaleza se desvincula progresivamente de cualquier origen divino.» (p. 2). Además, para Pardo, A. desde una consideración antropológica clásica, argumenta que la «dignidad humana va ligada al modo de ser racional del hombre, que puede manifestarse o no, dependiendo de la circunstancia, y es el fundamento de la actitud de respeto ético hacia el hombre, sean cuales sean sus circunstancias». (2010, p. 90). 


\section{Metodología}

Los análisis planteados en este artículo corresponden a una investigación de carácter descriptivo y propositivo, que adopta un enfoque cualitativo. Esto significa que los hallazgos y los resultados obtenidos son producto de un ejercicio interpretativo. Como ejercicio interpretativo, la investigación se apoyó en estrategias para la clasificación de la información, lo que requirió la evaluación de conceptos definidos previamente y la identificación de nuevas categorías que el análisis de la información sugirió para su comprensión. Para facilitar el análisis y recuperación de los datos clasificados, la información se codificó utilizando el programa ATLAS.ti versión 6.2.24. Se recurrió a la teoría fundamentada como perspectiva metodológica para clasificar y agrupar la información en unidades analizables. Aunque el propósito de la Teoría fundamentada es la construcción de teoría, en esta revisión documental no se pretendió llegar a la formulación de apartados teóricos debido a que no es el objetivo de la investigación. La investigación se desarrolló en tres etapas: Selección y búsqueda de información, Codificación y finalmente, Análisis y evaluación, en la tabla 1 se especifica la cantidad de textos analizados.

Tabla 1: Tipo y cantidad de textos analizados

\begin{tabular}{|c||c|c|c|c||c|c|}
\hline Tipo & Artículos & Libros & Ponencias & $\begin{array}{c}\text { Cap. } \\
\text { Libro }\end{array}$ & $\begin{array}{c}\text { Inf. de } \\
\text { Inv. }\end{array}$ & $\begin{array}{c}\text { Tesis } \\
\text { Doctoral }\end{array}$ \\
\hline \hline Cantidad & 76 & 9 & 8 & 7 & 3 & 2 \\
\hline
\end{tabular}

\section{Resultados en formación inicial del docente en ambientes b-learning}

Los retos para la formación de los docentes universitarios en ambientes b-learning se sustentan en un cambio de paradigma que se ubica en una posición equilibrada entre la educación presencial y la educación virtual. En otras palabras, la Educación b-learning, se encuentra arraigada en su dualismo entre 
actividades propuestas en la virtualidad (e-learning) y acciones presenciales donde se encuentran de manera sincrónica el docente y el estudiante. En este sentido, ésta debe privilegiar la formación del docente universitario en las dos dimensiones: la virtualidad y la docencia tradicional.

En principio, las habilidades y desempeños de los docentes en el manejo de la educación e-learning han sido resultados del aprender haciendo, de un interés por apropiarse en el conocimiento de plataformas (Moodle la más usada por ser software libre y Blackboard), como resultado de colocar en el ciberespacio sus cátedras para sus aprendices, quienes por otra parte, reciben algunas horas en el manejo de las aulas virtuales a manera de inducción a sus cursos y seminarios. Es decir, la educación e-learning en realidad se produce a partir de las experiencias de algunos docentes arriesgados en el uso de las Mediaciones Tecnológicas de la Información y Comunicación (MTIC) quienes se ven comprometidos con el manejo de cursos en esa modalidad.

Por otro lado, se registra una alta inversión de las Instituciones de Educación Superior (IES) en tecnología educativa, salas de informática, simuladores, bases de datos, plataformas. Por tal razón, el Ministerio de Educación Nacional, generó la normatividad específica (Ley 1188/2008, Decreto 1295 de 2010) donde se privilegian acciones para aumentar la cobertura y determinar las condiciones de calidad de los programas ofrecidos a los estudiantes colombianos que acuden a estos, para su formación en la educación superior. Es de aclarar, que la distinción entre la educación "a distancia, que permiten superar las limitaciones de distancia y tiempo y la educación virtual, que exigen el uso de redes telemáticas como entorno principal, hacen necesario el uso de las MTIC» (Padilla, 2011, p. 8), en tanto la educación a distancia y educación virtual, exige en lo referente a medios educativos, los escenarios de simulación virtual de experimentación y práctica, una plataforma tecnológica apropiada, la infraestructura de conec- 
tividad y las herramientas necesarias para su desarrollo (Decreto $1295,2010)$ que en últimas tiene que ser mediado por el docente en formación o en ejercicio.

En sentido amplio, se concibe el perfil del docente universitario para la educación b-learning como un «trabajador de conocimiento, diseñador de ambientes de aprendizaje, con capacidad para rentabilizar los diferentes espacios en donde se produce conocimiento» (Gros y Silva, 2005, p. 3); en otro aspecto, se propende por una formación integral del docente al esperar que «no sólo domine las didáctica específica de las diferentes materias sino también, una serie de elementos educativos transversales que todo profesional de educación debe adquirir» (Gros y Silva, 2005, p. 3), dejando tácitamente la adquisición de competencias y habilidades en el manejo de modelos pedagógicos, evaluación de los estudiantes, habilidades en multimedia, la elaboración de objetos de aprendizaje para lograr la comprensión de los contenidos, la dignidad humana y ética de sus estudiantes.

En la dimensión e-learning, se considera fundamental que en la «formación inicial y continua del profesorado incorpore en sus planes y acciones, la capacitación requerida para el diseño y tutorización de los entornos virtuales de aprendizaje» (Gros y Silva, 2005, p.12), la cual es complementada de una «formación suficiente como para saber qué tipo de modelo instruccional es más idóneo para adaptarlo a un contexto teleformativo» (Muñoz y González, 2010, p.1). Estos dos aspectos, uno complemento del otro, permiten la formación de un docente que no sólo está en condiciones para diseñar sus contenidos de aprendizaje sino, que además, se pregunta por el conocido modelo instruccional para el contexto. En realidad, algunos otros aspectos que complementan son los medios para la incorporación del material didáctico multimedial; la estructura de contenidos hipermedia y, otros más técnicos «como transformar la información en objetos de aprendizaje, cómo evaluar dichos materiales, cómo guiar, asesorar y orientar al 
alumnado a través de las herramientas sincrónicas y asincrónicas del LMS» (Muñoz y González, 2010, p.1).

Visto de esa manera, la formación inicial y continua del docente para ambientes b-learning debe contener «aspectos referentes al uso de lasi plataforma tecnológica y a innovadoras metodologías de enseñanza, utilizando tecnologías que capaciten para la innovación y renovación que los cambios tanto sociales, técnicos y científicos vayan requiriendo del papel del profesor» (González, 2007, p. 5). Adicionalmente, para lograr una transformación significativa del docente universitario «de transmisor de información a facilitador en la construcción del aprendizaje de los alumnos, es imprescindible la formación inicial y continua del profesorado tanto en el uso de herramientas tecnológicas, como de metodologías innovadoras y modelos de diseño instruccional que integren tecnologías de información y comunicación» (González, 2007, p. 2).

\section{Resultados en formación pedagógica del docente b-learning}

Una de las dimensiones fundamentales en la formación del docente que se desempeña en ambientes b-learning es la pedagógica, puesto que sus habilidades no se limitan solamente al manejo de la plataforma en la cual se desarrollan las acciones virtuales, sino que, por el contrario, se amerita que los ambientes de aprendizaje y la propuesta de actividades tanto grupales como individuales, tengan lineamientos y fundamentos en pedagogía y didáctica que garanticen el aprendizaje exitoso de los estudiantes y la comprensión de su realidad. Lo anterior se confirma con estudios afines donde se proponen «acciones de cualificación docente, no sólo en el uso tecnológico y operativo del aula virtual, sino además, en el campo pedagógico» (Padilla et ál., 2008, p. 4).

Estudios recientes del Grupo de Investigación PYDES realizados en Educación Superior (Padilla, 2011), (Padilla \& Páez, 2008) han 
demostrado que los docentes universitarios quienes trabajan en educación virtual, solicitan continuamente la formación pedagógica, didáctica y evaluativa para cualificar sus competencias en el manejo de las aulas y con mayor apuro, para los encuentros presenciales con los estudiantes. De igual forma, piensan que la orientación del aprendizaje, se deriva del conocimiento de estilos pedagógicos que se puedan aplicar en la educación virtual y a distancia. Algunos autores declaran que en Colombia «se ha venido avanzando no sólo en el ofrecimiento de programas virtuales sino en algunas transformaciones, particularmente en la formación de docentes y en los que podría denominarse como la nueva pedagogía con soporte virtual» (Alegre y Villar, 2006, p.16).

Así las cosas, el reto de formar pedagógicamente al docente universitario para que se desempeñe en ambientes b-learning, es de alto compromiso para las Facultades de Educación y los grupos que tienen como objeto de estudio este campo temático. En principio, porque se amerita «identificar los rasgos principales que caracterizan el aprendizaje del adulto» y «las responsabilidades de los tutores y alumnos» para el logro exitoso del aprendizaje (Didactic, 2011) y en últimas, porque la fundamentación pedagógica y didáctica le permite inferir y transformar algunos tipos de evaluación que ofrece la plataforma, caracterizados por ser principalmente de orientación conductista y denominadas como «herramientas de evaluación que posee una plataforma» (Didactic, 2011, p. 7). Mientras que hoy día, hay propuestas de formación docente en pedagogías contemporáneas para la educación a distancia y virtual (Padilla, 2011), que habilitan al docente en el conocimiento de perspectivas pedagógicas en Enseñanza para la Comprensión, Aprendizaje Autónomo, Aprendizaje Estratégico, Aprendizaje Basado en Problemas, Aprendizaje por Proyectos y Aprendizaje Colaborativo en función del conocimiento desde ambientes b-learning.

Para el desarrollo de habilidades y competencias evaluativas en el docente universitario en la educación ofrecida desde ambientes 
b-learning, se vislumbran dos escenarios: el presencial y el virtual que se complementan; puesto que el docente ha de definir su actuación para cada uno y, principalmente, establecer qué aprendizajes se evalúa en los estudiantes cuando se encuentra con sus pares de forma presencial y qué otros cuando está orientando las actividades propuestas desde el aula virtual que fortalezcan el desarrollo humano y social.

Por ejemplo, en el caso de los encuentros presenciales es necesario aprovecharlos en la sustentación de informes de investigaciones, discusión de problemáticas en las áreas de aprendizaje, acuerdos sobre acciones a seguir para la resolución de problemas donde se apliquen los conocimientos aprehendidos y, principalmente, en la comprensión de su realidad y la formación de competencias comunicativas y argumentativas entre los estudiantes. Todos los aspectos anteriores susceptibles de ser evaluados cualitativa y formativamente.

Mientras que en la formación docente para la educación e-learning se ha centrado en «conocer y saber aplicar estrategias didácticas... de colaboración y construcción [...] de contenido [...] que le permitan fomentar el desarrollo de habilidades y la interactividad de los aprendices» (Didactic, 2011, p. 6). Estudios más puntuales, dejan conocer que se incrementa "aprender a redactar comentarios de evaluación de las tareas realizadas por los alumnos bajo un enfoque formativo» (Didactic, 2011, p. 7) y en el rol de tutor se privilegia la importancia de tener una capacidad «en discernir competencias y criterios evaluativos en el marco de la evaluación que generará el docente en medios e-learning» (Didactic, 2011, p. 6). Pero, żqué significa evaluar desde un enfoque formativo? Para resolver la pregunta ha de capacitarse al docente en teorías evaluativas, comunicativas y de una posición crítica reflexiva que le lleve a la comprensión del acto educativo, como horizonte de formación de sus aprendices donde se privilegie la dignidad, el desarrollo humano y social. 


\section{Resultados en formación continua del docente para ambientes b-learning}

Al considerar hoy día la educación de la persona se encuentran conceptos como: educación para la vida, educación en la vida, educación para el trabajo y el desarrollo humano, y educación integral, entre otros. Pero, ipuede un docente universitario formado en ambientes b-learning dejar de actualizarse o hacer a un lado su capacitación continúa? Con el alto desarrollo de las Mediaciones de Información y Comunicación y, en complemento, con el auge de la actualización de las plataformas, que colaboraron con la educación e-learning y, además, a partir de la continúa reflexión desde las diferentes perspectivas pedagógicas, realizadas por los grupos de investigación a través de estudios en educación y pedagogía, invitan a los docentes a mantenerse actualizados de estos avances de forma constante. En consecuencia, se produce una gran cantidad de conocimiento acerca del saber específico de la educación a distancia y virtual que hace necesaria la educación continua del maestro ya sea en formación o en ejercicio. Ahora bien, en condiciones de aprendizaje $b$-learning, donde se complementan tanto el avance tecnológico como la adquisición de competencias pedagógicas y didácticas, se hace absolutamente necesaria la formación continua del talento humano docente, si las Instituciones de Educación Superior manifiestan querer estar al día en tecnología y en la oferta de programas profesionales de alta calidad y pertinencia.

Es decir, por una parte se plantea privilegiarse la formación tecnológica del docente en cuanto al uso adecuado de las herramientas ofrecidas por la plataforma que sirve de medio para la educación e-learning y, por otra parte, se hace prioritaria la formación de competencias pedagógicas que garanticen al docente el conocimiento y la experticia en propuestas para la orientación del aprendizaje de sus estudiantes y el desarrollo humano, para lograr en definitiva un uso pedagógico de las 
mediaciones tecnológicas de la información y comunicación. Por esta razón, se «hace necesario formar profesionales que deseen aprender y seguir aprendiendo continuamente, especialmente mientras recorren el fértil terreno de la vida profesional [...] e invertir en su capacidad para enfrentar futuro y la mejora de sus aptitudes colectivas» (Centro Interuniversitario de Desarrollo [CINDA], p. 214).

\section{Resultados en formación en la administración tecnológica para ambientes b-learning}

En relación con la administración de las plataformas que hacen posible el ofrecimiento de la educación a través de las aulas virtuales, conocida como e- learning, se presentan distintas extensiones en la formación del docente como: la gestión educativa, el diseño instruccional de aula, conocimientos de los aspectos técnicos, el manejo de textos y contenidos relacionados con el saber específico, el uso de herramientas Web 2.0 y Web 3.0, la apropiación de los diferentes sistemas evaluativos y la elaboración de informes parciales y definitivos de los estudiantes. De ahí que se han encontrado, publicaciones que hacen énfasis en formación del tutor-facilitador, del docente en aspectos técnicos y en el desarrollo de contenidos. Con miras a la formación docente hacia una educación humanista, es necesaria la fijación en un desempeño del maestro en: el diseño instruccional del aula, autoría de los contenidos y una propuesta pedagógica.

\section{Resultados en formación del tutor facilitador}

Aunque en algunos estudios en educación e-learning y b-learning aceptan distintos términos para el docente como: facilitador, tutor, orientador, en el presente trabajo se asigna el concepto de «tutor facilitador» para proponer los distintos roles que se le asignan al docente, quien atiende desde modalidades de educación en $b$-learning. Hasta ahora, se «asocia la docencia tradicional con 
el ejercicio del docente como TRANSMISOR (en algunos casos de información y en otros de conocimiento), en contraposición con la docencia b-learning y el rol de docente como FACILITADOR» (González, 2007, p. 2). Entonces, el reto de la formación del docente se presenta en un cambio substancial de tradicionaltransmisor a facilitador b-learning, puesto que se educa en ambientes tanto en e-learning como en presencial. Se detectó, además, la necesidad «de formar docentes flexibles al cambio que dejen de ser transmisores de información y pasen a ser facilitadores en la construcción del aprendizaje de los alumnos» (Dellepiane, 2010, p. 9). En síntesis, la formación del docente podría determinar un cambio en el rol, en cuanto pasa de ser un transmisor a un facilitador de los procesos de aprendizaje con un alto fortalecimiento en su dimensión humana y de significativa responsabilidad social.

$\mathrm{Al}$ preguntar si existen investigaciones que relaten los temas y las áreas de formación para el tutor facilitador en ambientes b-learning, se encuentran estudios que sugieren aspectos como: «evaluación (conceptos, procedimientos e instrumentos), basada en competencias, con métodos distintos en pruebas de conocimiento a través de herramientas y métodos: portafolio, wikis, plataformas de trabajo colaborativo (BSCW) y LMS». (Zapata, 2010, p. 8). Pero queda por definir si el docente ha de recibir formación en selección y evaluación de diseños instruccionales para dictaminar su conveniencia, que a la vez se traduce en metaevaluación de los procesos formativos, con base en el criterio de eficiencia del diseño en los medios empleados por el formador. Se puede apreciar que se hace énfasis sobre la formación evaluativa, teniendo en cuenta los aspectos metodológicos y la formación en competencias de los estudiantes, dejando a un lado la especificidad en la formación humana y la responsabilidad social. Pero, ante esta realidad, es necesario que en los docentes se desarrollen «destrezas suficientes no sólo para la utilización y diseño de los medios, sino para su 
selección y evaluación» (Méndez et ál., 2007, p. 42), lo cual haría posible el surgimiento de un docente crítico y reflexivo al servicio de la educación desde ambientes b-learning, consciente de su acción humanista y de alta sensibilidad por las problemáticas sociales.

Por este motivo, se espera la acreditación de «Títulos académicos, publicaciones e investigaciones que demuestren la capacidad del docente para acompañar en el proceso de aprendizaje y que aporten conocimientos y retos al estudiante para que éste, a través de la interacción con los materiales, redes de conocimiento, las bases de datos bibliográficas y sus compañeros, pueda lograr los objetivos de aprendizaje trazados para el curso» (Velásquez, 2009, p. 2), lo cual hace necesaria la formación de un docente desde un horizonte humano, ético, complejo, interactivo, fundamentado en la selección y análisis de la información y, especialmente, con alta fundamentación pedagógica y humanística.

\section{Resultados en formación docente en los aspectos técnicos}

En cuanto al manejo exitoso de las plataformas que son herramientas utilizadas como medio para la educación b-learning, se han realizado estudios que demuestran alguna experticia de tipo empírico, resultado del ensayo y error de los docentes y lejos de ser efecto de capacitación directa e intencionada, Padilla, J. E. (2008). Para el docente universitario que continuamente maneja plataformas como Moodle o Blackboard, por ejemplo, en principio han llegado a niveles favorables de suficiencia en la utilización desde el aprendizaje autónomo y producto de su interés por el manejo de las plataformas; Padilla, J. E. (2011). Por lo general, se pretende lograr que en «la formación en entornos virtuales haya una conceptualización acerca de qué es una plataforma y las diversas características técnicas y tecnológicas» (Alegre, 2010). Esto permite, a su vez, «aprender a organizar la información que 
nos proporciona la plataforma tecnológica de forma que podamos realizar el seguimiento de los alumnos en un curso» (Didactic, 2011, p. 3) y, en consecuencia, desarrollar habilidades en el manejo de herramientas para la evaluación del aprendizaje y la retroalimentación a los estudiantes, tema principal en el avivamiento de la participación estudiantil y el logro de su desarrollo humano y su contribución con el progreso de la sociedad.

Por otra parte, el tutor facilitador regularmente se encuentra ante el dilema del manejo de sus actividades a desempeñar. Lo que hace necesario «conocer las funciones que debe desarrollar el tutor e-learning y diferenciar los estilos de aprendizaje que diferencian los autores»(Didactic, 2011, p. 7). En esta línea, aspectos como la organización de la información suministrada por la plataforma $y$, en consecuencia, llevar a cabo la retroalimentación del estudiante, amerita una experticia adecuada por el tutor facilitador quien potencia sus aportes cuando realiza «la creación de carpetas de los estudiantes, evalúa desde un enfoque formativo y utiliza adecuadamente las herramientas para la evaluación ofrecida por la plataforma» (Didactic, 2011, p. 7). Al momento, de acuerdo con (Gros y Silva, 2005, p. 9) se potencia el uso de las redes sociales para lo cual es necesario que el docente conozca qué tipos de redes existen como Facebook, MySpace, Tuenti, los mundos virtuales especialmente como Second Life, su definición, características, experiencias formativas y usos didácticos, que sirven como apoyo a la educación e-learning y que fortalecen en los estudiantes la comprensión en la realidad.

En general, la formación en el manejo de plataformas para el docente tiene dos aspectos igualmente importantes: lo tecnológico, conformado por todo el paquete que ofrece la plataforma y el diseño instruccional consistente en la adecuada presentación y manejo de contenidos de su saber donde se asienta el sentido humano y social. 


\section{Resultados de formación en diseño instruccional para el docente en b-learning}

En los cursos ofrecidos en ambientes b-learning, donde se esperan desempeños de los docentes tanto para las actividades presenciales, como para las ofrecidas a través de las MTIC o en algunos casos desde el Aula Virtual solamente, quedan aspectos por formar en el docente como es el caso del diseño instruccional, la creación de materiales, las actividades sincrónicas y asincrónicas, el tipo de contenidos, la construcción textual, la comunicación y la selección de la información que se sube al aula, para el logro de los aprendizajes de los educandos. Es decir, que gran parte de la formación docente ha estado orientada a la educación ya sea presencial o a la virtual, mediante actividades e-learning, pero queda el reto de establecer una sólida formación en diseño instruccional para cursos ofrecidos en b-learning, con la perspectiva de un desarrollo humano en el estudiante.

En principio, la gran ventaja que presenta la educación a través del e-learning es la posibilidad para acceder a actividades en el momento que el estudiante lo estime conveniente y, además, que no es absolutamente necesario asistir físicamente a un lugar determinado, haciendo preciso que el tutor «conozca qué es la comunicación asincrónica así como sus ventajas e inconvenientes» (Didactic, 2011, p. 8), lo cual implica que el docente deberá adquirir habilidades tales como procesos comunicativos e informáticos para establecer canales de interacción con el estudiante. De igual forma, la comunicación sincrónica permite la interacción de los estudiantes en actividades como el chat y que amerita del docente «conocer las funciones que debe desarrollar el tutor en los foros de discusión, moderar los debates y realizar la retroalimentación a los estudiantes» (Didactic, 2011, p. 7) y que vienen siendo actividades análogas con las conocidas mesas redondas en la educación presencial pero se distancian en la medida que son realizadas mediante el uso de las MTIC. 
De otro lado, respecto a los contenidos en el desarrollo de las temáticas propuestas por el curso, donde el reto del tutorfacilitador es conocer un sinnúmero de fuentes de información, relacionarlas con los objetivos del curso, que le permitan al estudiante el análisis y la reflexión sobre problemáticas en el área de trabajo y, además, lo contextualice en el campo profesional y laboral. En este orden de ideas, los contenidos depositados en el aula están lejos de ser bancos de información y búsqueda digital, por el contrario, se asume que tienen sentido y pertinencia al ser tan finamente escogidos que el estudiante los encuentra asertivos y complementarios en su formación como futuro profesional; retos en la formación docente como diseño del material de apoyo, el manejo de la multimedia, el uso adecuado tanto de la hipermedia como las herramientas sincrónicas y asincrónicas, las estrategias de participación y la representación estética del aula hacen que se siga estudiando por los grupos de investigación aspectos centrales del diseño instruccional en la modalidad $b$-learning.

\section{Conclusiones}

Se hace prioritaria la formación del docente en cuanto a la dignidad humana, la ética mundial, en el uso de alternativas como elearning (educación electrónica) y b-learning («blended learning» o aprendizaje combinado), el diseño y administración de aulas virtuales, uso de herramientas Web 2.0 y 3.0, la autoría de textos de su asignatura, manejo de software especializado de su área $y$, principalmente, un conocimiento de perspectivas pedagógicas y estrategias didácticas que le permitan el diseño de ambientes de aprendizaje para sus aprendices, acordes al contexto y a los problemas sociales de la comunidad.

La ética y la moral van cambiado de acuerdo a los tiempos, de acuerdo a las construcciones socioculturales, políticas y en mayor presunción educativas, las cuales atañen a un sujeto interdisci- 
plinar, metacognitivo y dinámico en tiempos donde los flujos de información hacen que el docente y su formación, transformen su proceso pedagógico con veras a establecer un marco conductual y aptitudinal basado en la preservación de los principios y valores, protegiendo y resguardando un patrimonio de principios de tolerancia y protección del entorno. Sea cual sea el campo de interacción, su acción de formación, más aún en educación superior, debe fortalecer en el educando una prospectiva de convivencia sobre la paulatinas transformaciones demarcadas en el contexto de la era digital.

El devenir profesional del docente en relación con el uso de las MTIC, hace sumamente relevante un proceso formativo referente a la ética y el dilema de la implementación y beneficio de las tecnologías comunicativas, en tanto se respeten los derechos de autor, las licencias otorgadas para el beneficio educativo y la formación para establecer políticas de uso en el tipo de software y plataformas, más en el contexto b-learning, donde el paradigma de facilitación del aprendizaje, otorga al docente, una responsabilidad frente a transmitir a sus estudiantes un sentido de uso adecuado de las MTIC.

La formación integral del docente en el contexto b-learning, debe promover en los profesionales un sentido de pertenencia y acción social para formar y formarse en términos de un desempeño idóneo, con bases en la educación a partir de la comprensión, al hacer uso del escenario social como epicentro de análisis y así extrapolar situaciones problémicas en la reflexión disciplinar y científica; sin dejar de lado el hecho de los principios éticos y humanos que en sí, establecen lógicas de comunicación y tolerancia, incentivando la subjetividad del ser en su inmersión social.

Un modelo humanista, además de los ya enunciados desde la combinación de espacios presenciales y virtuales, se justifica, en la medida de promover desde la interacción, la comunicación y la 
reflexión con los estudiantes, una sensibilización de los procesos formativos sin dejar de lado la construcción de sujeto, a partir del desarrollo sostenible, adjudicado por las dinámicas sociales con base en los juicios, leyes y acciones de orden axiológico, encausadas por las problemáticas no sólo inferidas de las ciencias humanas, también las que competen el criterio profesional en la educación del docente en cualquier área disciplinar.

La formación inicial, pedagógica, continúa, en administración de TIC y tutorización, procesos de crecimiento y superación profesional de suma importancia en el contexto b-learning, debido a que el docente se enfrenta a los transcursos de masificación, estandarización y autorregulación, cuyo fin hacen más compleja su labor. Por tal razón, tendrá que estar en una marcha constante de aprendizaje para la especialización en MTIC, metodologías y estrategias concernientes a una interacción basada en el paradigma metacognitivo. Igualmente, contemplar y entender los medios y contextos del estudiante, para aprender y consolidar distintas maneras de formarse desde los estilos de aprendizaje; una opción de comprender al estudiante como un promotor de su propio saber.

\section{Bibliografía}

Alegre, M. (2010). Capacidades docentes para atender la diversidad. Una propuesta vinculada a las competencias básicas. Revista Educación Inclusiva, 3 (3).

Alegre, M. y Villar, L. (2006). Evaluación de la formación en línea del profesorado de cinco Universidades Españolas. Revista de Universidad y Sociedad del Conocimiento, 1 (4), 1-19.

Centro Interuniversitario de Desarrollo (CINDA), (2000). Las nuevas demandas del desempeño profesional y sus implicancias para la docencia universitaria. Santiago: Gestión Universitaria. 
Dellepiane, P. (2010). Diseño de contenidos virtuales: experiencia en la materia Turismo Sustentable de la Licenciatura en Turismo y Hotelería de UADE. Congreso Iberoamericano de Educación: Metas 2021. Universidad Argentina.

Didactic. (2011). Formación para profesionales del E-learning. Recuperado de http://www.avalnavirtual.com/didactic/

González, J. (2007). Blended learning, un modelo pertinente para la educación superior en la sociedad del conocimiento. Recuperado de http://ihm.ccadet.unam.mx/virtualeduca2007/ pdf/95-JGM.pdf

Gros, B. y Silva, J. (2005). La formación del profesorado como docente en los espacios virtuales de aprendizaje. Revista Iberoamericana de Educación 36 (1). 1-13.

Küng, H. (2008). Ética mundial en América Latina. Madrid: Trotta, pp. $67-78$

López, M. (2006) Una filosofía humanista de la educación. México: Trillas.

Méndez, A., Rivas, A. y Del Toro, M. (2007). Entornos virtuales de Enseñanza Aprendizaje. Ministerio de Educación Superior, Editorial Universitaria.

Muñoz, P. y González, M. (2010). Formación del profesorado universitario en el uso y manejo de herramientas multimedia bajo sistemas e-learning. Revista Iberoamericana de Educación $52(5), 1-14$.

Padilla, J. E. (2008). Globalización y educación: un desafío en la formación del docente universitario. Bogotá: Editorial Bonaventuriaa.

Padilla, J. E. (2008). La formación del docente universitario con miras al desarrollo humano. Revista Educación y Desarrollo Social, Bogotá: pp.90-99. 
Padilla, J. E. (2011). La formación docente par el uso de las mediaciones tecnológicas de información y comunicación en: Revista Educación y Desarrollo Social, 5, 1, p. 8.

Padilla, J. E. y González, K. (2010). Análisis de los estilos de aprendizaje a través de la implementación de actividades a través de una Wiki. Medellín, Antioquía: Revista Virtual, Universidad Católica del Norte.

Padilla, J. E., Páez, C. y Montoya, R. (2008). Creencias de los docentes acerca del uso de las tecnologías de información y comunicación. Revista Educación y Desarrollo Social 2(2) , 45-47.

Pardo, A. (2010). Cuestiones básicas de Bioética. Madrid: Rial, $122 \mathrm{p}$.

Porlan, R. y Martí. J. (1996). El diario del profesor: un recurso para la investigación en el aula, Sevilla, España: Diada Editora.

Pelé, A. (2005). Una aproximación al concepto de dignidad humana. Universidad Carlos III de Madrid: Revista Universitas, 1. p. 9-13.

Velásquez, O. (2009). El nuevo rol del docente virtual para entornos virtuales de aprendizaje, «el caso coipa». Recuperado de http:// mail.ceipa.edu.co/ ceipa/lupa/ediciones_online/1030705.pdf

Zapata, M. (2010). Evaluación de competencias en entornos virtuales de aprendizaje y docencia universitaria. Revista de Educación a Distancia, 1, 2-34.

Recibido $\epsilon$ abril de ZOII Arbitrado $\in$ n junio de 2Oll 